\title{
Evidence for the Universal Scaling Behaviour of a Freely Relaxing DNA Molecule
}

\section{Citation}

Manneville, S., Philippe Cluzel, J.-L. Viovy, D. Chatenay, and F. Caron. 1996. Evidence for the universal scaling behaviour of a freely relaxing DNA molecule. Europhysics Letters 36, no. 6: 413-418.

\section{Published Version}

http://dx.doi.org/10.1209/epl/i1996-00244-6

\section{Permanent link}

http://nrs.harvard.edu/urn-3:HUL.InstRepos:3645425

\section{Terms of Use}

This article was downloaded from Harvard University's DASH repository, and is made available under the terms and conditions applicable to Other Posted Material, as set forth at http:// nrs.harvard.edu/urn-3:HUL.InstRepos:dash.current.terms-of-use\#LAA

\section{Share Your Story}

The Harvard community has made this article openly available.

Please share how this access benefits you. Submit a story.

\section{Accessibility}




\title{
Evidence for the universal scaling behaviour of a freely relaxing DNA molecule
}

\author{
S. Manneville ${ }^{1}$, Ph. Cluzel ${ }^{1}\left(^{*}\right)$, J.-L. Viovy ${ }^{1}$, D. Chatenay ${ }^{2}$ and F. Caron ${ }^{3}$ \\ 1 Laboratoire PCC (UMR CNRS 168), Institut Curie, Section de Recherche \\ 11, rue P. et M. Curie, 75231 Paris, France \\ ${ }^{2}$ LUDFC, Institut de Physique - 3, rue de l'Université, 67084 Strasbourg, France \\ 3 Ecole Normale Supérieure, Laboratoire de Genetique Moleculaire \\ 46, rue d'Ulm, 75230 Paris, France
}

(received 5 February 1996; accepted 10 October 1996)

PACS. 36.20-r - Macromolecules and polymer molecules.

PACS. 87.15-v - Molecular biophysics.

\begin{abstract}
Relaxation measurements on a fluorescently labelled free DNA molecule after stretching by a Poiseuille flow in a capillary vessel reveal universal scaling features: at intermediate times the scaling exponent of the decay law for the molecule length as a function of time is found to be $0.51 \pm 0.05$. This law is in agreement with the prediction of the Brochard-Wyart "stem and flower" model for the relaxation of a stretched polymer chain.
\end{abstract}

Introduction. - Real-time imaging of single DNA molecules in solution by fluorescence microscopy has provided a new approach to the investigation of polymer dynamics [1]. Previous experimental observations on tethered molecules [2], [3] combined with various applied problems related to the deformation of "polymer brushes" have promoted the development of new theoretical models to describe a single polymer chain under strong flows [4], [5]. The relaxation of a chain after stretching, in particular, has been the object of several recent studies. Perkins et al. [2] investigated the relaxation of a single DNA molecule attached to a bead maintained in an optical tweezer, after elongation in a flow. They observed that the terminal relaxation time scales as $L^{1.68}$, in rather good agreement with the Zimm model. Later on, Brochard proposed a "stem and flower" model for the earlier stages of relaxation, in which the entropic contribution to the chain free energy is much larger than $k T$, and the Zimm or Rouse models are not expected to be valid. In this letter we investigate the relaxation of free DNA molecules after elongation in a flow, thus avoiding any potential problem associated with beads or tweezers. We focus on the influence of the chain length and of the solvent viscosity on the relaxation process.

Theoretical background. - The theoretical description of a tethered polymer chain under flow distinguishes between three regimes depending on the flow velocity: at low flows, the chain can be modelled as a weakly deformed coil (the end-to-end distance is of the order of the radius of gyration $R_{\mathrm{g}}$ ) (see, e.g., the calculation by Zimm quoted in [3]). A model based on the "blob" picture was recently proposed by Brochard [5] in order to describe tethered chains at higher flow velocities: above a critical flow velocity, the chain unwinds partly and is

$\left({ }^{*}\right)$ Present address: Princeton University, Physics Department, Princeton, 08542 NJ, USA. 


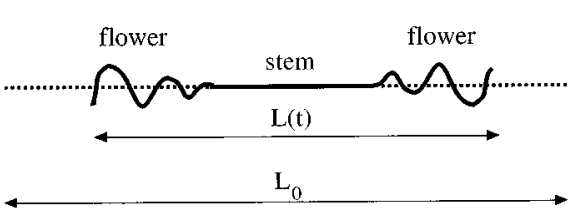

Fig. 1.

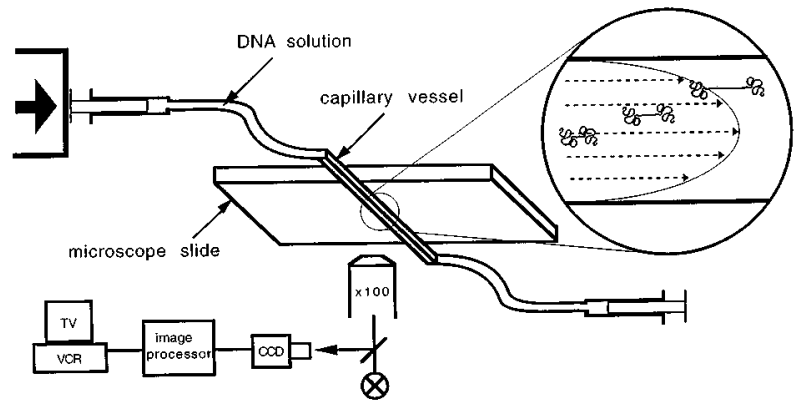

Fig. 2.

Fig. 1. - The "stem and flower" conformation of a free polymer chain relaxing by its two ends.

Fig. 2. - Experimental scheme.

composed of a stretched portion, the "stem", ending in a fluctuating coil, the "flower" (fig. 1). By increasing the flow further, the flower finally disappears and the chain is considered as fully stretched. If the flow is stopped, the chain relaxes by its free end, adopting at intermediate times a "stem and flower" conformation again [6].

For an ideal chain (Flory exponent $\nu=1 / 2$ ), the end-to-end distance $L_{\mathrm{f}}$ of the flower growing at the free end of the retracting stem scales with time $t$ as

$$
L_{\mathrm{f}}(t) \propto\left(\frac{k T}{\eta a} t\right)^{1 / 2}\left(\ln \left(\frac{k T}{\eta a^{3}} t\right)\right)^{1 / 2}
$$

( $k$ is the Boltzmann constant, $T$ the absolute temperature, $\eta$ the solvent viscosity, and $a$ the persistence length; all numerical coefficients are ignored).

For a chain with excluded volume $(\nu=3 / 5)$, the prediction yields

$$
L_{\mathrm{f}}(t) \propto\left(\frac{k T}{\eta a} t\right)^{1 / 2} .
$$

For most practically important situations (and in particular for the present study $\left({ }^{1}\right)$ ) the variation of the logarithmic term in eq. (1) is small and can be neglected, leading to a power law dependence for either ideal or swollen chains.

The stem was shown to shrink with the same time dependence, i.e.

$$
L_{0}-L_{\mathrm{s}} \propto L_{\mathrm{f}}(t) \propto\left(\frac{k T}{\eta a} t\right)^{1 / 2}
$$

where $L_{\mathrm{s}}$ is the length of the stem and $L_{0}$ the initial length of the molecule. This yields for the total length $L=L_{\mathrm{f}}+L_{\mathrm{s}}$

$$
L_{0}-L_{\mathrm{f}}(t) \propto\left(\frac{k T}{\eta a} t\right)^{1 / 2}
$$

$\left({ }^{1}\right)$ Experimentally, $a \cong 50 \mathrm{~nm}$ and $\eta$ ranges between $30 \mathrm{cP}$ and $180 \mathrm{cP}$, so that $\tau_{0}=\eta a^{3} / k T \cong 1 \mathrm{~ms}$. Time $t$ varies from about $0.5 \mathrm{~s}$ (minimal time necessary to stop the flow to about 10 to $100 \mathrm{~s}$ ). Therefore, $t / \tau_{0}$ varies by less than two orders of magnitude around $10^{3}$ to $10^{4}$, so that $\ln \left(t / \tau_{0}\right)$ remains roughly constant during the relaxation process. 


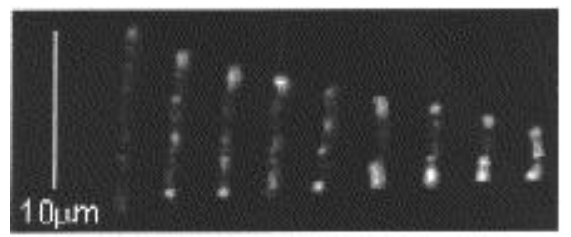

Fig. 3. - Relaxation of a single $\lambda$-phage DNA molecule. Frames are spaced at $3 \mathrm{~s}$ intervals. The first frame corresponds to time $t=0.5 \mathrm{~s}$ after the flow arrest.

When the chain length has decreased to a few $R_{\mathrm{g}}$, the "stem and flower" description of the chain is no longer valid and the chain enters a different relaxation regime that will not be considered in this paper $\left({ }^{2}\right)$.

Materials and methods. - We used dilute solutions of wild-type $\lambda$-phage DNA (48502 bp) in a phosphate buffer $(0.1 \mathrm{M})$ of $p \mathrm{H}$ 7.4. In such conditions the persistence length of DNA is about $50 \mathrm{~nm}$ and the radius of gyration about $0.7 \mu \mathrm{m}$ [7], [8]. Shorter molecules (38416 bp and 24508 bp referred to by $3 / 4 \lambda$ and $1 / 2 \lambda$ ) were obtained by enzymatic digestion by restriction enzymes Xba I and Apa I, respectively (New England Biolabs) followed by phenol extraction and ethanol precipitation.

Fluorescent staining was performed with YOYO-1 (Molecular Probes, Inc.) [9]: a DNA solution at $1 \mu \mathrm{g} / \mathrm{ml}$ and a $0.5 \mu \mathrm{M}$ YOYO-1 solution were mixed at 1:1 ratio (one YOYO-1 molecule per five base pairs). The stained DNA solution was mixed with glycerol in various proportions for obtaining viscosities ranging from $30 \mathrm{cP}$ to $180 \mathrm{cP}$ and $4 \%$ (vol.) $\beta$-mercaptoethanol was added to reduce photobleaching [1]. The solution was placed in a rectangular capillary vessel $50 \times 500 \mu \mathrm{m}$ (Vitro Dynamics, Inc.) and visualized at room temperature $(T=295 \pm 2 \mathrm{~K})$ using a Zeiss Axiovert 135 inverted epifluorescence microscope with a $\times 100$ Plan-Neofluar objective of numerical aperture 1.3. By pushing the solution with a syringe pump through the capillary, a Poiseuille flow was created that stretched the DNA perpendicular to the plane of observation [10]. This plane was located at half the distance between the center of the capillary and the capillary wall to obtain efficient stretching and avoid wall effects. The flow velocity in the center of the capillary ranged from 100 to $500 \mu \mathrm{m} \mathrm{s}^{-1}$ (Reynolds number $\cong 10^{-4} \ll 1$ ), corresponding to a force variation of the order of one piconewton $(\mathrm{pN})$ over the size of the statistical coil. The flow in the capillary was stopped in about $0.5 \mathrm{~s}$ using another syringe at the other end of the tubing. Images of individual molecules at $0.04 \mathrm{~s}$ intervals were intensified by a Hamamatsu MCP image intensifier, recorded by a CCD camera (Cohu, Inc.), and processed by a Hamamatsu Argus 10 image processor (fig. 2). The accuracy of one length measurement was $0.3 \mu \mathrm{m}$ and the reproducibility from one measurement to another was of the order of $1 \mu \mathrm{m}$.

Experimental results and discussion. - Video images of the relaxation of a free DNA molecule after the flow arrest show the growth of two fluctuating coils at each end of a rapidly shrinking rod portion (fig. 3). In order to restrict our analysis to the region relevant to scaling analysis, we measured $L(t)$ until no rod portion could clearly be seen. For a $\lambda$-phage DNA molecule, this corresponds to $L(t) \cong 4 \mu \mathrm{m}$. The initial lengths averaged during $1 \mathrm{~s}$ before the flow arrest was $L_{0}=16.1 \pm 0.3,12.6 \pm 0.3$, and $8.1 \pm 0.3 \mu \mathrm{m}$, respectively, for the $\lambda$,

$\left({ }^{2}\right)$ In the present experiment we investigated the relaxation of a free chain and not that of a tethered chain. However, apart from the terminal relaxation mode, a free chain of initial length $L_{0}$ is equivalent to two tethered chains of length $L_{0} / 2$ attached together. Thus, the scaling in eq. (4) also holds for a free chain relaxing by its two ends in the "stem and flower" regime. 


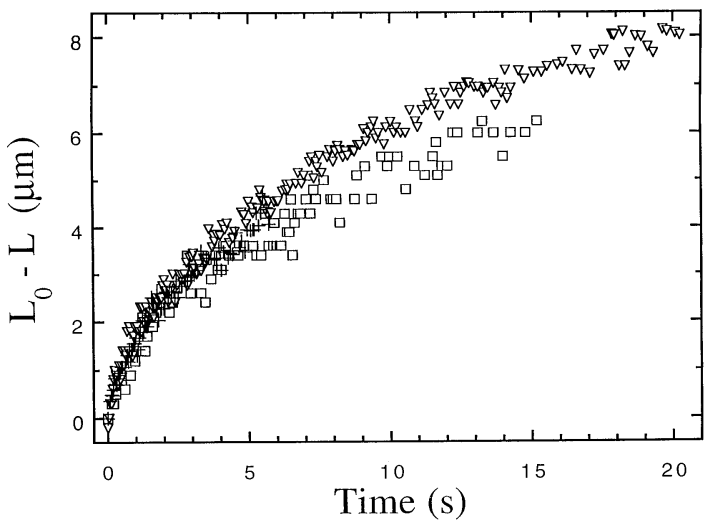

Fig. 4.

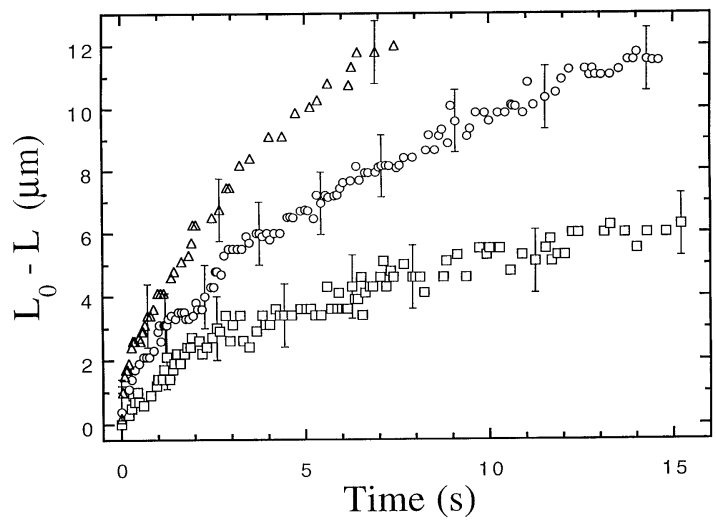

Fig. 5.

Fig. 4. - Relaxation of single free DNA molecules: $\lambda$ (squares), $3 / 4 \lambda$ (crosses), and $1 / 2 \lambda$ (triangles). Data points before time $t=0$ are used to measure the experimental initial lengths $L_{0}=16.1 \mu \mathrm{m}$, $12.6 \mu \mathrm{m}$, and $8.1 \mu \mathrm{m}$, respectively (see text).

Fig. 5. - Relaxation of free $\lambda$-phage DNA molecules in solvent of viscosities $\eta \cong 37 \mathrm{cP}$ (triangles), $70 \mathrm{cP}$ (circles), and $170 \mathrm{cP}$ (squares).

$3 / 4 \lambda$, and $1 / 2 \lambda$ molecules. The commonly accepted contour lengths (obtained, e.g., when the molecule is adsorbed on a surface or fully aligned in a crystal) $\left({ }^{3}\right)$, however, are $20.3,16.0$, and $10.2 \mu \mathrm{m}$, respectively. Therefore, although these molecules visually presented no apparent coil at the ends, their length under shear was smaller than the static contour length. A similar behaviour was obtained by Perkins et al., see [2], [3]. This point deserves some comments since it questions the meaning of the "contour length" for a molecule in thermal equilibrium in a fluid. The force-extension curve of DNA has a high slope in the vicinity of the crystallographic length $L_{\mathrm{c}}$ [8], but it does not diverge and indeed the molecule can be extended far beyond $L_{\mathrm{c}}$, progressively mobilizing the degrees of freedom [11], [12] with higher energies, such as bending on scales smaller than the persistence length, torsion of bond angles, and rearrangements of the base pairs. The different sections of a chain in strong flow do not experience the same force, so that they do not mobilize the same degrees of freedom: a rigorous description of its extension should take into account all of them. In particular, note that all models for the relaxation of a tethered chain proposed to date implicitly rely on a bead-spring or freely jointed chain model. It was shown in [8] that the force-extension curve of DNA deviates strongly from the FJC prediction for extensions larger than $0.75 L_{\mathrm{c}}$ (i.e. forces larger than about $0.5 \mathrm{pN}$ ), and that the behaviour at lower extensions could be modelled by an "equivalent FJC chain" with a contour length $L_{\mathrm{fjc}} \cong 0.8 L_{\mathrm{c}}$, rather than $L_{\mathrm{c}}$. Since DNA in our experiments experiences initial forces of the order of $1 \mathrm{pN}$, the initial length we measured is consistent with the behaviour reported in [8], and confirms that the observed relaxation should be relevant to the "stem and flower" regime. To remain consistent with this model, however, we used the length $L_{\mathrm{fjc}}$ as the initial length, i.e. $16.2,12.8$, and $8.2 \mu \mathrm{m}$ for the $\lambda, 3 / 4 \lambda$, and $1 / 2 \lambda$ molecules, respectively (for the reasons discussed above, in our stretching conditions these lengths happen to be equal to the observed initial length within experimental error).

$\left({ }^{3}\right)$ From [1], [3], [8], labelling at saturation with ethidium bromide (EB), which corresponds to about one EB per two bp, yields an increase in length of about $30 \%$. Using our labelling ratio of one bis-intercalator for five bp, and assuming that the variation of the rise per base pair induced by one YOYO dimer is comparable to that induced by two EB, we expect an increase in the contour length of $23 \%$ as compared with naked DNA. 


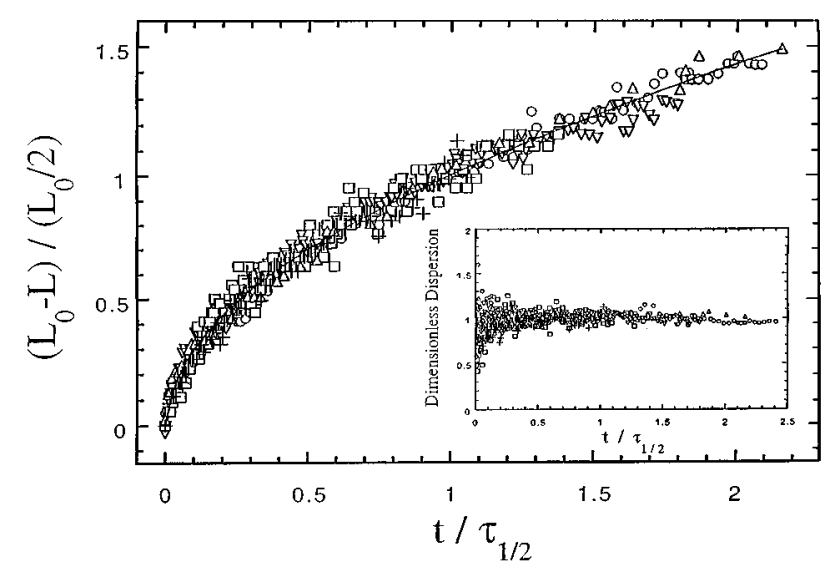

Fig. 6. - Rescaling analysis of the relaxation data from fig. 4 and 5. The power law fit (solid line) yields a scaling exponent of 0.51 . Inset: dispersion around the theoretical prediction (ratio of $\left(L_{0}-L(t)\right) /\left(L_{0} / 2\right)$ by $\left(t / \tau_{1 / 2}\right)^{1 / 2}$ as a function of $\left.t / \tau_{1 / 2}\right)$.

Figure 4 displays the relaxation of free DNA molecules of different lengths $L_{\mathrm{fjc}}$ at a given solvent viscosity $(\eta \cong 170 \mathrm{cP})$. The data show that in the same solvent and within our precision of $0.3 \mu \mathrm{m}$ on $L(t)$ and $L_{0}$, the different fragments undergo the same relaxation process at short times. Some deviations of about $1 \mu \mathrm{m}$ are observed in the late stages of the relaxation either between experiments on molecules of different sizes or between experiments performed at different times on molecules of the same size. These deviations represent the typical experiment-to-experiment reproducibility of our measurements. Within this experimental uncertainty, the relaxation process is independent of the initial length (i.e. of the number of monomers in the chain) for a given solvent viscosity and temperature. This first experimental result is in agreement with the "stem and flower" model (eq. (4)).

The relaxation of free $\lambda$-phage DNA molecules $\left(L_{\mathrm{fjc}}=16.2 \mu \mathrm{m}\right)$ was then measured for three different solvent viscosities: $\eta \cong 37,70$ and $170 \mathrm{cP}$ (fig. 5). To rescale the data, we measured the "half-relaxation time" $\tau_{1 / 2}$ (given by $\left.L\left(\tau_{1 / 2}\right)=L_{0} / 2\right)$ and plotted $\left(L_{0}-L(t)\right) /\left(L_{0} / 2\right.$ ) as a function of $t / \tau_{1 / 2}$ (fig. 6). Note that this rescaling method is model-independent, has no arbitrary component, and does not require the exact value of the solvent viscosity, since $\tau_{1 / 2}$ is experimentally well defined for each data set. The rescaled data collapsed on a single, universal curve (fig. 6) and were fitted to a power law, yielding a scaling exponent of 0.51 (average over the relaxations of different molecules in various conditions; independent fits yielded exponents ranging from 0.48 to 0.57 without any systematic dependence on chain length or solvent viscosity).

We checked that the uncertainty on the flow arrest time did not affect the results significantly, by letting the origin of times as a fitting parameter: the fitted value was within $0.2 \mathrm{~s}$ of the experimental one and the exponent did not change by more than 0.01 . Because $L_{\mathrm{fjc}}$ is not as well defined a quantity as $L_{\mathrm{c}}$, we also varied this parameter by $\pm 1 \mu \mathrm{m}$ and the exponent changed to 0.52 and 0.49 , respectively. Finally, although the onset of relaxation visually seems rather sharp (see, e.g., fig. 4), the relevance of the first data points could be questioned because of possible non-instantaneous arrest of the flow and because the "stem and flower" model is not expected to be valid at very short times. However, removing the first $0.25 \mathrm{~s}$ or the first $0.5 \mathrm{~s}$ from the data sets only modified the average exponent to 0.50 and 0.49 , respectively. Figure 6 (inset) shows that the ratio of $\left(L_{0}-L(t)\right) /\left(L_{0} / 2\right)$ by $\left(t / \tau_{1 / 2}\right)^{1 / 2}$ as a function of $t / \tau_{1 / 2}$ remains constant and equal to 1 within less than $10 \%$ for $t / \tau_{1 / 2}>0.2$. As expected, larger deviations are obtained at short times due to the limited temporal and spatial resolution. 
We also performed the fit with truncations of the late stages of relaxation varying from $80 \%$ to $20 \%$ of the full $L_{\mathrm{fjc}}$. The fitted exponent remained within the error range of 0.05 with little or no systematic trend, supporting the idea that this exponent really corresponds to a relaxation regime and not to a fortuitous effect of the truncation of data imposed by experiment. Finally, we also fitted various raw data from [2] using the same protocol as for our data, i.e. fitting the relaxed length $L_{\mathrm{fjc}}-L(t)$ to a power law in $t$ with $L_{\mathrm{fjc}}=0.8 L_{\mathrm{c}}$, and truncating the data for $L(t)<0.2 L_{\mathrm{fjc}}$. Slight deviations appeared at very short times, but the fit to the remainder of the data was correct and yielded scaling exponents in the range 0.43-0.52. We conclude that the relaxation of a tethered chain is essentially equivalent to the relaxation of one half of the free chain, and is well described by the "stem and flower" model except for the terminal range: the discrepancy previously quoted in [2] was probably due to the inclusion of this terminal relaxation range, not relevant to the model, in the fit.

Conclusions. - The relaxation of free DNA chains extended in a shear flow after flow arrest follows the scaling behaviour predicted by Brochard's "stem and flower" scaling model in the range in which this model is expected to apply. It is worth noticing that this range is limited: under forces higher than typically $0.5 \mathrm{pN}$, the force-extension behaviour of DNA departs from the freely jointed chain prediction, and no model for early stages of relaxation is presently available. However, from the shape of the force-extension curve [8], we anticipate that these strong forces relax within a few fractions of a second. The "stem and flower" model is also irrelevant for the terminal relaxation process, when the flowers interact hydrodynamically and a Zimm-like terminal time is expected [2]. This limited range is the consequence of the large persitence length of DNA: even a large chain does not have as many statistical segments as one would wish for comfortably studying universal behaviour. Nevertheless we believe that the present study, using several viscosities and molecular sizes, provides a convincing demonstration of the validity of the "stem and flower" model.

We particularly thank Prof. PH. Akar for his technical help, T. T. PERkins for communicating to us the data tables from ref. [2] and for fruitful discussions about the experiment, and Prof. F. BRochard-Wyart for helpful discussions on theoretical questions.

\section{REFERENCES}

[1] Houseal T. W., Bustamante C., Stump R. F. and Maestre M. F., Biophys. J., 56 (1989) 507.

[2] Perkins T. T., Quake S. R., Smith D. E. and Chu S., Science, 264 (1994) 822.

[3] Perkins T. T., Smith D. E., Larson R. G. and Chu S., Science, 268 (1995) 83.

[4] Adjari A., Brochard-Wyart F., de Gennes P.-G., Leibler L., Viovy J.-L. and RubinSTEIN M., Physica A, 204 (1994) 17.

[5] Brochard-Wyart F., Europhys. Lett., 23 (1993) 105.

[6] Brochard-Wyart F., Europhys. Lett., 30 (1995) 387.

[7] Frontali C., Dore E., Ferrauto A. and Gratton E., Biopolymers, 18 (1979) 1353.

[8] Smith S. B., Finzi L. and Bustamante C., Science, 258 (1992) 1122.

[9] Rye H. S., Yue S., Wemmer D., Quesada M., Haugland R., Mathies R. and Glazer A., Nucleic Acids Res., 20 (1992) 2803.

[10] Barrat J.-L., Macromolecules, 25 (1992) 832.

[11] Cluzel P., Lebrun A., Heller C., Lavery R., Viovy J.-L., Chatenay D. and Caron F., Science, 271 (1996) 792.

[12] Marko J. and Siggia E. B., Macromolecules, 28 (1995) 8759. 